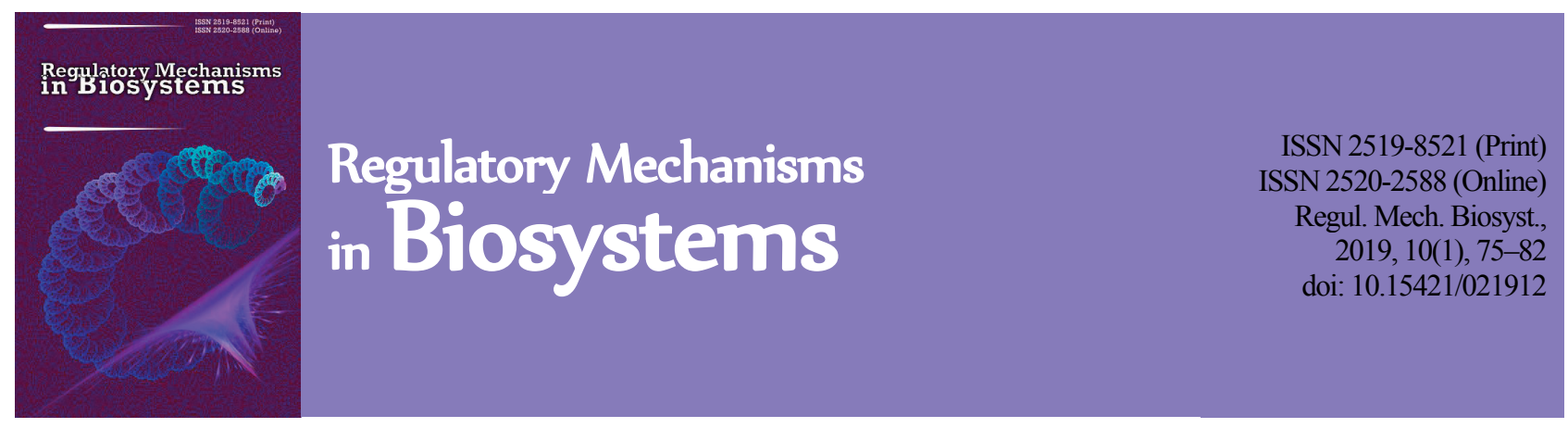

\title{
Synergistic and antagonistic effects of insecticide binary mixtures against house flies (Musca domestica)
}

\author{
M. A. Levchenko, E. A. Silivanova \\ All-Russian Scientific Research Institute of Veterinary Entomology and Arachnology, \\ Branch of Federal State Institution Federal Research Centre Tyumen Scientific Centre, Siberian Branch \\ of the Russian Academy of Sciences (ASRIVEA - Branch of Tyumen Scientific Centre SB RAS), Tyumen, Russian Federation
}

Article info

Received 15.01.2019

Received in revised form 20.02.2019

Accepted 22.02.2019

All-Russian Scientific Research Institute of Veterinary Entomology and Arachnology, Tyumen Scientific Centre SB RAS, Institutskaya st., 2 Tyumen, 625041, Russia. Tel.: + 7-345-225-85-58. E-mail:11eas@vniivea.ru, easylva@gmail.com

\section{Levchenko, M. A., \& Silivanova, E. A. (2019). Synergistic and antagonistic effects of insecticide binary mixtures against house flies (Musca domestica). Regulatory Mechanisms in Biosystems, 10(1), 75-82. doi:10.15421/021912}

The house fly, Musca domestica Linnaeus, 1758 (Diptera, Muscidae), is known as a globally distributed parasite with veterinary and medical importance and the ability to develop resistance to insecticides Insecticide mixtures can contribute to enhancing the effectiveness of existing insecticides against house flies and to implementing insecticide resistance management. The present study was conducted to assess the efficacy of four insecticides with different modes of action, applied alone and in binary mixtures, against adults of the $M$. domestica laboratory strain by no-choice feeding bioassays. The interaction patterns of neonicotinoid acetamiprid, phenylpyrazole fipronil, avermectin ivermectin, and pyrrole chlorfenapyr in the binary mixtures were likewise analyzed by calculating the combination indices to find out combinations with the synergistic effect. The analysis of values of insecticide lethal concentrations for 50\% mortality revealed that the toxicity of acetamiprid, fipronil, and ivermectin increased in the binary mixtures compared to when they applied alone, while the toxicity of chlorfenapyr depended on the second insecticide in the mixtures. The combination index values of five insecticide mixtures, fipronil/acetamiprid (1:10), fipronil/chlorfenapyr (1:4), ivermectin/acetamiprid (1:2.5), ivermectin/chlorfenapyr (1:3 and 1:10) were $<1$, which displays a synergism. Three insecticide mixtures, acetamiprid/chlorfenapyr (1:4), fipronil/ivermectin (1:4), fipronil/chlorfenapyr (1:40), had combination index values $>1$, which indicates an antagonism. The fipronil/chlorfenapyr (1:4) mixture was the more toxic to adults of $M$. domestica. The ivermectin/chlorfenapyr (1:10) mixture and the ivermectin/acetamiprid (1:2.5) mixture produced the highest synergistic effects. The results of the present study suggest that the interaction patterns (synergistic or antagonistic) in the insecticide mixtures can depend on both the combination of insecticides and their ratio. Further studies are required in order to evaluate the synergistic combinations against field populations of $M$. domestica.

Keywords: combination index; feeding test; insecticide interaction; insecticide mixture; house fly control.

\section{Introduction}

The house fly, Musca domestica Linnaeus, 1758, is a common pest inhabiting animal and poultry farms and urban areas across the world. M. domestica is known as a vector of more than 100 animal and human pathogens (Malik et al., 2007; Zhu et al., 2016), including bacteria, viruses, fungi, protozoans, helminth eggs (Wang et al., 2011; Doud et al., 2014; Khamesipour et al., 2018). Participation of flies in the spread of antibiotic resistance microorganisms has been assessed as well (Usui et al., 2015; Mohammed et al., 2016; Nazari et al., 2017). Thus, the house fly has veterinary and medical importance. Insecticides are the most effective tools for insect control;however, the house fly is able to develop resistance to insecticides (Khan et al., 2013a; Scott et al., 2013). Researchers from different countries have reported $M$. domestica field populations with resistance to such insecticides as organochlorides, organophosphates, carbamates, pyrethroids, neonicotinoids, spinosyns,ciromazine, and others (Kaufman et al., 2001; Acevedo et al., 2009; Markussen \& Kristensen, 2011; Khan et al., 2013a; Abbas et al., 2015). According to Zhu et al. (2016), $M$. domestica has exhibited documented resistance to 62 insecticide active ingredients.

Insecticide resistance is an actual and serious problem in pest control (Bass et al., 2015), and resistance management is important and crucial for effective insect control in agriculture and in public health (Sparks \& Nauen, 2015). Several strategies have been described for insecticide resistance management (Sudo et al., 2017). The use of insecticide mixtures has been recognized as an effective anti-resistance strategy along with the use of rotation or mosaics of insecticides (Durel et al., 2015; Sudo et al., 2017). In theory, insecticide mixtures with synergistic effect may reduce the insecticidal load on the environment as well (Ritz \&Streibig, 2014). Logically, active ingredients with different modes of action are the appropriate candidates to prepare mixtures due to their ability to complement one another and due to a minimal probability of development of resistance to two different ingredients simultaneously in insects.

Neonicotinoids, including acetamiprid, are nicotinic acetylcholine receptor agonists (Casida \& Durkin, 2013; Sparks \& Nauen, 2015) and are known as relatively quick neuroactive insecticides. Phenylpyrazole fipronil belongs to antagonists of $\gamma$-aminobutyric acid (GABA) gated chloride channels (Casida \& Durkin, 2013) and has a slower insecticide effect than acetamiprid. Avermectins are activators of chloride ion channels (Sparks \& Nauen, 2015), in particular, ivermectin acts via glutamategated chloride channels in parasites (Omura \& Crump, 2004). While acetamiprid, fipronil, and ivermectin are neurotoxins, pyrrole chlorfenapyr affects insects' metabolism because it is an oxidative phosphorylation uncoupler (Black et al., 1994; Sparks \& Nauen, 2015). Taking into account the different modes of action of the listed insecticides, they could be applied in mixtures.

Mixtures of commonly used pyrethroids and organophosphates have been evaluated against many insects including Culex quinquefasciatus Say (Corbel et al., 2003), Spodoptera litura F. (Ahmad et al., 2009), Acyrthosiphon pisum Harris (Taillebois \& Thany, 2016), Plutella xylo- 
stella L. (Nasir et al., 2013) and M. domestica (Khan et al., 2013b). Combinations of these insecticides with phenylpyrazole fipronil or avermectins (like abamectin, emamectin) have been also studied against, for example, Anopheles gambiae (Ngufor et al., 2017), Frankliniella occidentalis (Cloyd \& Raudenbush, 2014), P. xylostella (Nasir et al., 2013) and M. domestica (Khan et al., 2013b; Abbas et al., 2015). Insecticidal effects of mixtures of new insecticides like chlorfenapyr have been reported against $A$. gambiae (Ngufor et al., 2016) and Culex pipiens pallens Coq (Yuan et al., 2015). There are no reports about toxicity of ivermectin or chlorfenapyr in combination with other insecticides against $M$. domestica. The present study was conducted to assess the efficacy of acetamiprid, fipronil, ivermectin, and chlorfenapyr, applied alone and in the binary mixture, against the house fly $M$. domestica by feeding tests. We analyzed as well the interaction patterns of these four insecticides in the binary mixtures to find out combinations with the synergistic effect that is of interest for effective applications of pesticides.

\section{Materials and methods}

Insects. Adults of the M. domestica laboratory strain were used to estimate the toxicity of insecticides under laboratory conditions. The laboratory strain of $M$. domestica was obtained from Novosibirsk Agrarian University (Russian Federation) in 2009 and was kept in the insectarium without contact with insecticides for more than 50 generations. Flies were reared at $26-28{ }^{\circ} \mathrm{C}, 50-60 \%$ relative humidity (RH) and $12: 12$ hours of light:dark photoperiod. The adult flies were kept in metal cages $(25 \times 25 \times 25 \mathrm{~cm})$, covered with a fine mesh. Rearing cages were supplied with water (cotton wicks in cups with water), and glucose and milk powder (1:1 by weight). Three to five-day old adult flies (without division by sex) were used in the tests.

Insecticides. Four insecticide active ingredients (as a technical substance) from different chemical classes were tested: acetamiprid (97\%, King Quenson Industry Group Ltd, China), ivermectin (97\%, Chengdu Newsun Biochemistry Co., Ltd, China), fipronil (99\%, King Quenson Industry Group Ltd, China), and chlorfenapyr ( $99 \%$, Chengdu Newsun Biochemistry Co. Ltd, China) (Table 1).

Table 1

Main characteristics of insecticides tested

Insecticide $\begin{gathered}\text { Chemical } \\ \text { class }\end{gathered}$

Note: modes of action of insecticides are according to Casida \& Durkin (2013) and Sparks \& Nauen (2015); chemical structures of insecticides were obtained from https://pubchem.ncbi.nlm.nih.gov.

Feeding bioassays. The toxicity of the insecticides was evaluated by no-choice feeding bioassays. Sugar cubes $(2-5 \mathrm{~g})$ were treated with aceto- ne solutions $(0.3 \mathrm{ml})$ of insecticides or insecticide mixtures. Each insecticide was tested at five to ten concentrations at least three times. The concentration of insecticides was expressed in $\mu \mathrm{g}$ of active ingredient per $g$ of sugar. In the control experiment, the sugar was treated with pure acetone $(0.3 \mathrm{ml})$. After the acetone evaporated, sugar cubes were placed in glass cups. Then twenty flies, which were starved for 12 hours prior to the experiment, were introduced into each cup. The cups were sealed with mesh pistons from the top and supplied with water drinkers (Fig. 1). The mortality of flies was recorded after 24 hours of exposure to insecticides.

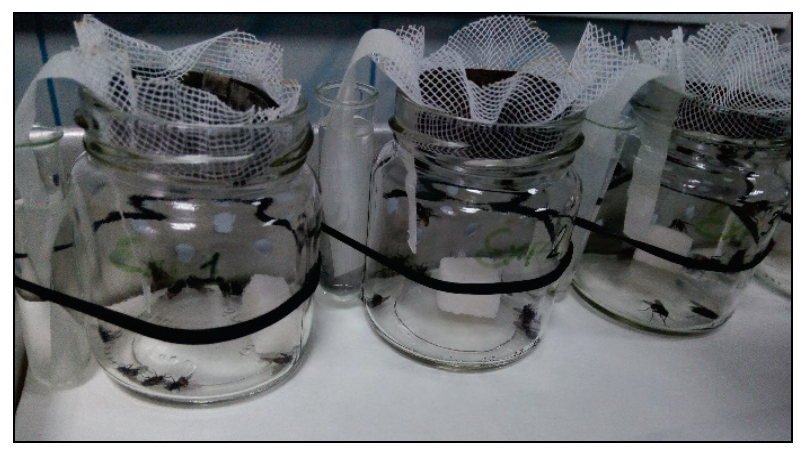

Fig. 1. Exposure of flies to sugar cubes treated with insecticides

Data analysis. Statistical analysis was performed using MedCalc Software version 18.2.1. The dose-response mortality in no-choice feeding bioassays was analyzed by probit regression analysis to calculate lethal concentrations for $50 \%\left(\mathrm{LC}_{50}\right)$ mortality for $95 \%$ confidence interval and to find slope values of probability-log dose regression lines. Interaction pattern (synergistic, additive and antagonistic) of insecticides tested alone and in the mixture were analyzed by the combination index (CI) (Khan et al., 2013b), that was calculated for $50 \%$ mortality by the formula:

$$
C I_{50}=\frac{L C_{50} A m}{L C_{50} A}+\frac{L C_{50} B m}{L C_{50} B}+\left(\frac{L C_{50} A m}{L C_{50} A} \times \frac{L C_{50} B m}{L C_{50} B}\right)
$$

In this formula, $\mathrm{LC}_{50} \mathrm{~A}$ and $\mathrm{LC}_{50} \mathrm{~B}$ are median lethal concentrations of insecticide $\mathrm{A}$ and $\mathrm{B}$ respectively, when tested alone, and $\mathrm{LC}_{50} \mathrm{Am}$ and $\mathrm{LC}_{50} \mathrm{Bm}$ are their median lethal concentrations when tested in the mixture. The interaction pattern of insecticides in the mixture was considered as an additive when combination index $=1$, as antagonistic when combination index $>1$, and as synergistic when combination index $<1$ (Khan et al., 2013b).

Each concentration of insecticides was tested at least three times. Difference between median lethal concentrations of insecticide was considered statistically significant when $95 \%$ confidence intervals were non-overlapping.

\section{Results}

The toxicity of four active substances to adults $M$. domestica of the laboratory strain, based on $\mathrm{LC}_{50}$ values, increased in the order acetamiprid $<$ ivermectin $\leq$ chlorfenapyr $<$ fipronil (Table 2 ). The slope value for fipronil was the lowest; the slope of the regression line was less steep compared to other active substances.

Changes in the toxicity of active substances in mixtures compared to when they applied separately are shown in Figures 2-5. Based on dose-mortality plots (Fig. 2-4) and $\mathrm{LC}_{50}$ values (Table 2,3) of each of the active substances, the acetamiprid toxicity increased and the fipronil and ivermectin toxicities slightly increased in all mixtures compared to when applied alone. The chlorfenapyr toxicity decreased in the mixture with acetamiprid, remained the same in the mixture with fipronil (at the fipronil/chlorfenapyr ratio 1:40), and increased in the remaining mixtures (Fig. 5). The combination of chlorfenapyr with each of the three insecticides resulted in a decrease in their $\mathrm{LC}_{50}$ values in the mixtures. The same effect was observed for ivermectin and fipronil (except the fipronil/chlorfenapyr mixture at ratio 1:40). The addition of acetamiprid to fipronil or ivermectin led to decrease in their $\mathrm{LC}_{50}$ values, and contrarily increased the $\mathrm{LC}_{50}$ value of chlorfenapyr (Table 3). 
Table 2

Toxicity of four insecticides, when applied alone and in binary mixtures, to adults of Musca domestica in the laboratory no-choice feeding bioassay

\begin{tabular}{lccccc}
\hline \multicolumn{1}{c}{ Insecticide } & Ratio & $\begin{array}{c}\text { Number } \\
\text { of flies }\end{array}$ & $\begin{array}{c}\mathrm{LC}_{50}(95 \% \text { confidence } \\
\text { interval }), \mu \mathrm{g} / \mathrm{g} \text { of sugar }\end{array}$ & $\mathrm{x} \pm \mathrm{SE}$ \\
\hline acetamiprid & $1: 0$ & 680 & $14.207(10.410-19.181)^{\mathrm{d}}$ & $1.599 \pm 0.105$ \\
fipronil & $1: 0$ & 460 & $0.156(0.095-0.257)^{\mathrm{c}}$ & $0.959 \pm 0.099$ \\
ivermectin & $1: 0$ & 540 & $1.077(0.347-45.621)^{\mathrm{abd}}$ & $1.335 \pm 0.118$ \\
chlorfenapyr & $1: 0$ & 680 & $3.909(2.320-8.075)^{\mathrm{b}}$ & $1.551 \pm 0.112$ \\
acetamiprid + chlorfenapyr $1: 4.0$ & 500 & $7.878(4.336-14.315)^{\mathrm{d}}$ & $0.744 \pm 0.058$ \\
fipronil + acetamiprid & $1: 10.0$ & 780 & $1.021(0.632-1.649)^{\mathrm{a}}$ & $0.695 \pm 0.041$ \\
fipronil + ivermectin & $1: 4.0$ & 800 & $0.636(0.170-2.082)^{\mathrm{ac}}$ & $1.691 \pm 0.101$ \\
fipronil + chlorfenapyr & $1: 4.0$ & 480 & $0.400(0.216-0.738)^{\mathrm{ac}}$ & $0.615 \pm 0.050$ \\
fipronil + chlorfenapyr & $1: 40.0$ & 650 & $3.303(2.890-3.761)^{\mathrm{b}}$ & $2.706 \pm 0.193$ \\
ivermectin + acetamiprid $1: 2.5$ & 460 & $1.151(0.653-2.031)^{\mathrm{a}}$ & $0.867 \pm 0.068$ \\
ivermectin + chlorfenapyr $1: 3.0$ & 700 & $1.196(0.710-2.015)^{\mathrm{a}}$ & $0.705 \pm 0.045$ \\
ivermectin + chlorfenapyr 1:10.0 & 360 & $0.752(0.273-2.073)^{\mathrm{a}}$ & $0.775 \pm 0.091$ \\
\hline
\end{tabular}

Note: $\mathrm{LC}_{50}$ : lethal concentrations for $50 \%$ mortality of flies; values with the same letter have not statistically significant difference (confidence intervals are overlapping); slope corresponds to $b$ coefficient in the equation of the regression line by probit analysis and reflects the rate of change in the probability of the flies' mortality with an increase in the dose of insecticide; $\mathrm{x}$ - slope.

For the mixtures, their toxicity to flies increased in the order acetamiprid/chlorfenapyr $<$ fipronil/chlorfenapyr $(1: 40)<$ ivermectin/acetamiprid $=$ fipronil/acetamiprid $=$ ivermectin/chlorfenapyr $(1: 3)<$ fipronil/ivermectin $=$ ivermectin/chlorfenapyr $(1: 10)<$ fipronil/chlorfenapyr (1:4). The acetamiprid/chlorfenapyr mixture was significantly less toxic
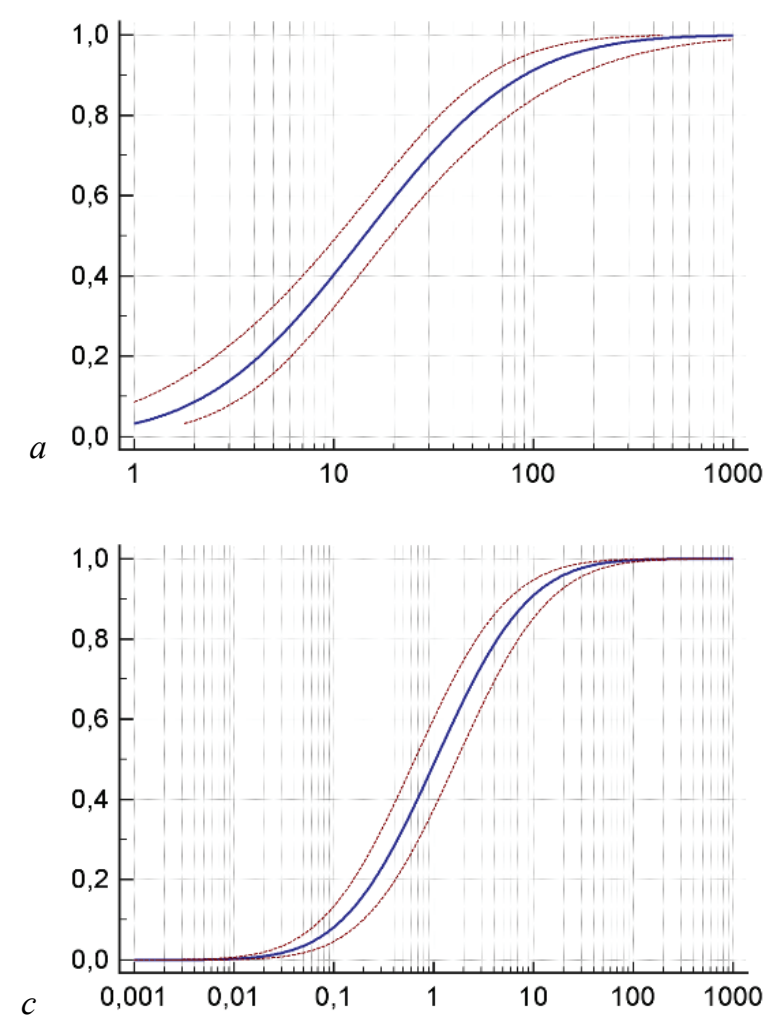

(non-overlapping $95 \%$ confidence interval for $\mathrm{LC}_{50}$ ) than the other mixtures. The insecticide ratio in the ivermectin/chlorfenapyr mixtures had no significant effect on their toxicity, based on dose-response plots (Fig. $6 g-h$ ) and $\mathrm{LC}_{50}$ values of these mixtures (Table 2). The toxicity of the fipronil/chlorfenapyr mixture was significantly higher at the 1:4 than that at the 1:40 ratio. As can be seen in Figure $6 c$ and $6 d$, the toxicity of the fipronil/chlorfenapyr mixture at the 1:40 ratio increased more rapidly compared with that at the ratio $1: 4$ in the dose interval $1-10 \mu \mathrm{g} / \mathrm{g}$ of sugar. This is consistent with the slope values in Table 2. The slope of the regression line for the fipronil/chlorfenapyr mixture at the 1:40 ratio was 4.4-fold higher than at the 1:4 ratio. In addition, the slope values of the fipronil/chlorfenapyr (1:40) and fipronil/ivermectin mixtures were greater than those of other mixtures, which indicates a steeper increase in the toxicity of these mixtures per unit dose. The slopes of regression lines for other mixtures were no different (Table 2).

Table 3

Combination index $(\mathrm{CI})$ values of insecticide mixtures against adults of Musca domestica by the laboratory no-choice feeding bioassay

\begin{tabular}{llccc}
\hline Insecticide mixture (A+B) & Ratio & $\mathrm{LC}_{50} \mathrm{~A}$ & $\mathrm{LC}_{50} \mathrm{~B}$ & $\mathrm{CI}$ (for $\mathrm{LC}_{50}$ ) \\
\hline acetamiprid + chlorfenapyr & $1: 4.0$ & 2.190 & 8.759 & 2.74 \\
fipronil + acetamiprid & $1: 10.0$ & 0.099 & 0.988 & 0.75 \\
fipronil + ivermectin & $1: 4.0$ & 0.127 & 0.509 & 1.67 \\
fipronil + chlorfenapyr & $1: 4.0$ & 0.099 & 0.397 & 0.80 \\
fipronil + chlorfenapyr & $1: 40.0$ & 0.081 & 3.223 & 1.77 \\
ivermectin + acetamiprid & $1: 2.5$ & 0.420 & 1.051 & 0.49 \\
ivermectin + chlorfenapyr & $1: 3.0$ & 0.434 & 1.302 & 0.87 \\
ivermectin + chlorfenapyr & $1: 10.0$ & 0.068 & 0.683 & 0.25 \\
\hline
\end{tabular}
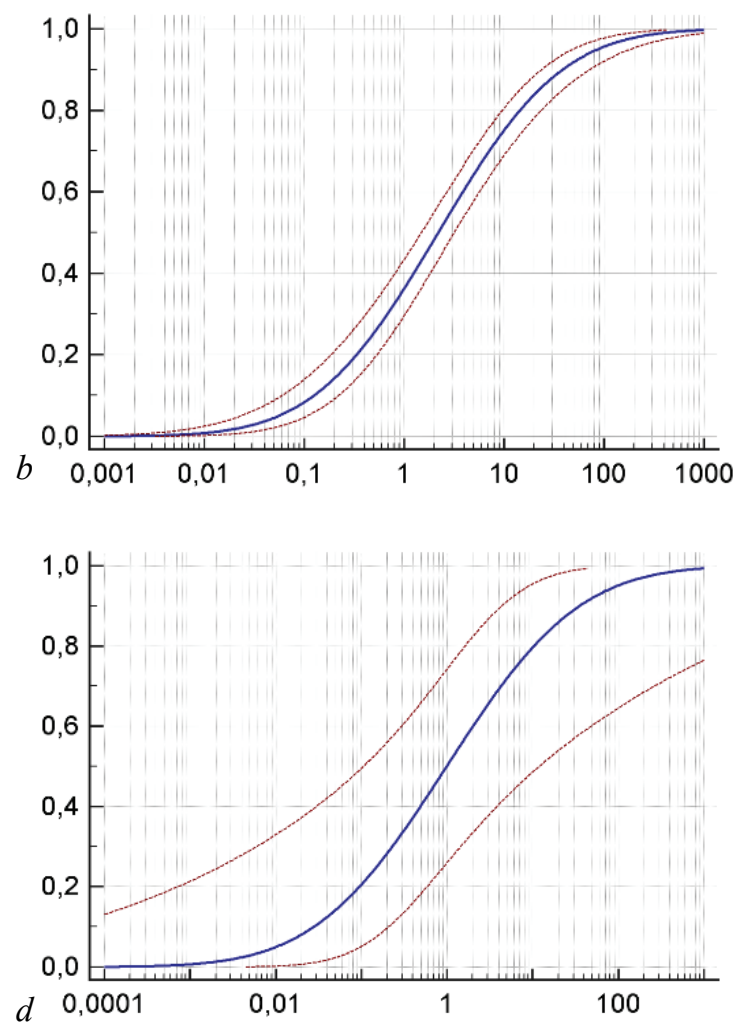

Fig. 2. Dose-response plots (blue line) with confidence intervals (red lines) for acetamiprid tested alone ( $a$ ) and in the acetamiprid/chlorfenapyr $(b)$, ivermectin/acetamiprid (c), and fipronil/acetamiprid (d) mixtures against adults of Musca domestica in the laboratory no-choice feeding bioassay:abscissae (horizontal) are doses ( $\mu \mathrm{g} / \mathrm{g}$ of sugar), ordinates (vertical) are probabilities of fly mortality

Three of tested mixtures had antagonistic effect with combination index values $>1$ (Table 3 ). The highest antagonistic effect was observed for the acetamiprid/chlorfenapyr mixture. The mixtures of fipronil with ivermectin or chlorfenapyr (1:40) were antagonistic as well. Five of the tested mixtures revealed synergistic effect with combination index values $<1$ (Table 3 ). The highest synergistic effect was observed for the ivermectin/chlorfenapyr mixtures at the 1:10 ratio; however, the effect of the ivermectin/chlorfenapyr combination at the 1:3 ratio was close to the additive.

\section{Discussion}

The correct use of insecticides is one of the crucial factors that impact on the efficacy of insect control at livestock and poultry farms. Durelet al. 
(2015) noted the need to preserve the effectiveness of existing insecticides like synthetic pyrethroids against pests of livestock for as long as possible. The use of mixtures of existing insecticides can contribute to this. For house fly control, mixtures of pyrethroids with new insecticides such as fipronil, spinosad, emamectin were effective against susceptible and resistant field populations of house fly (Khan et al., 2013b; Abbas et al., 2015).
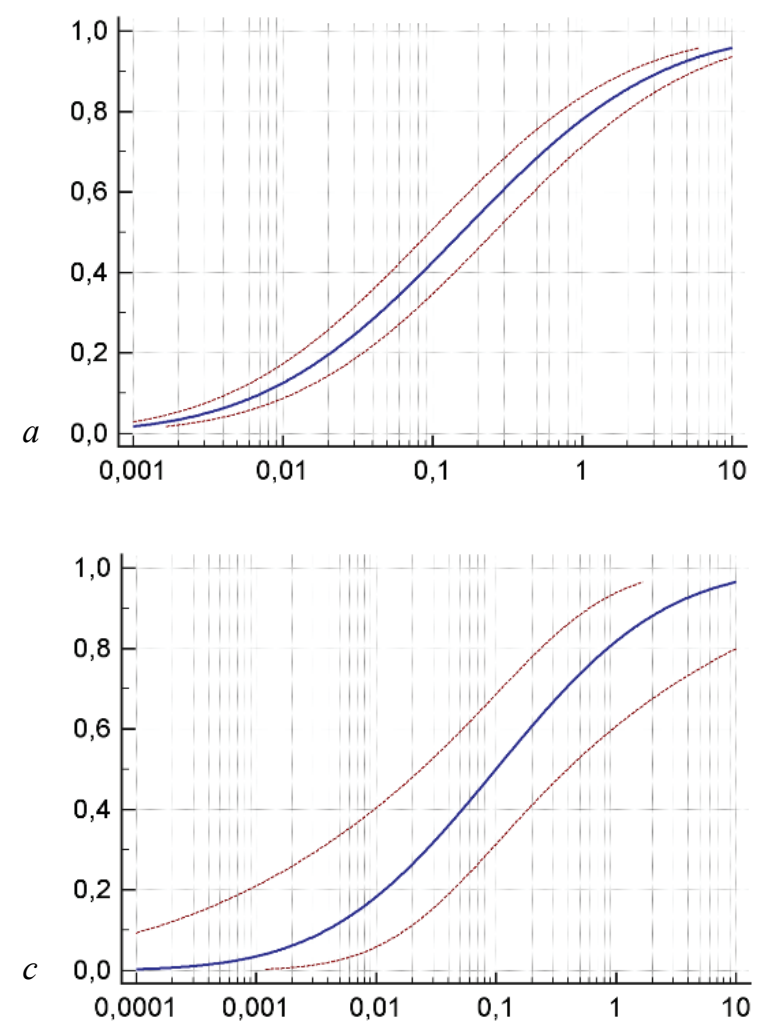

Along with pyrethroids, neonicotinoids have been widely used for house fly control at livestock and poultry facilities, and field populations of $M$. domestica resistant to neonicotinoids have been documented (Kaufman et al., 2010; Bass, 2015). In this context, the knowledge of which combinations of insecticides may have a synergistic or antagonistic effect would be useful for choosing the relevant insecticide treatment for effective fly management.
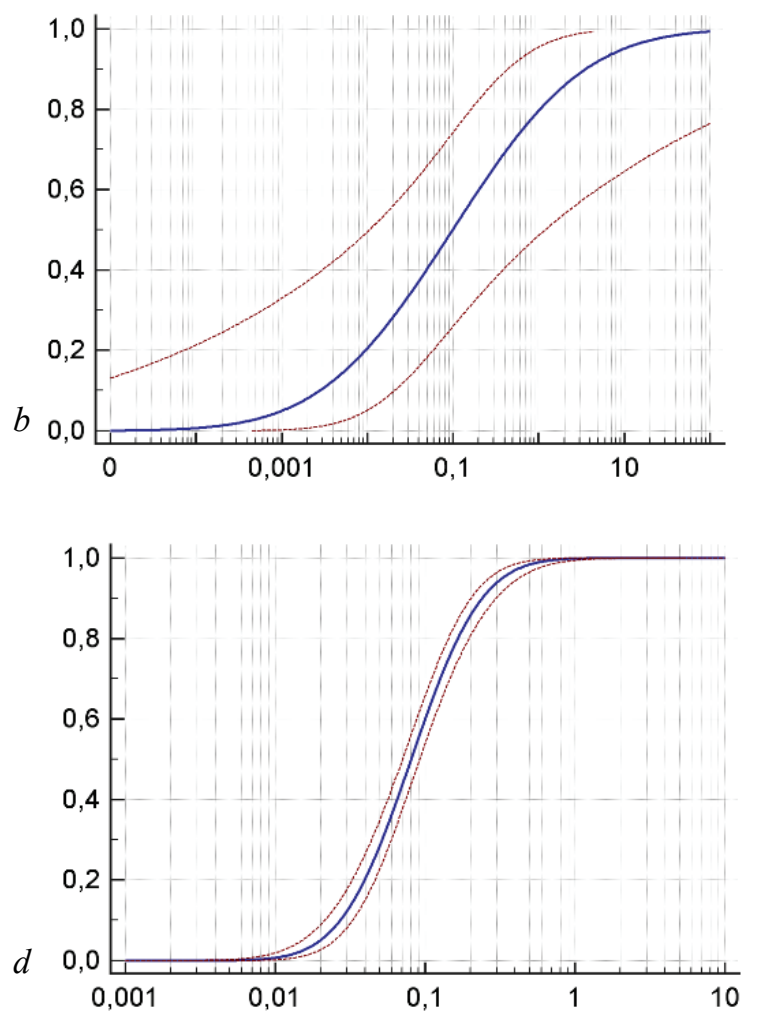

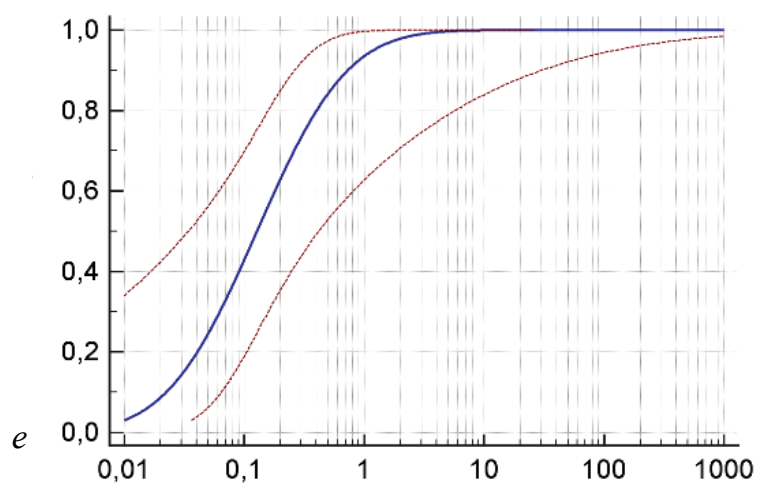

Fig. 3. Dose-response plots (blue line) with confidence intervals (red lines) for fipronil tested alone $(a)$ and in the fipronil/acetamiprid $(b)$, fipronil/chlorfenapyr (1:4) (c), fipronil/chlorfenapyr (1:40) $(d)$, and fipronil/ivermectin $(e)$ mixtures against adults of Musca domestica in the laboratory no-choice feeding bioassay: abscissae (horizontal) are doses ( $\mu \mathrm{g} / \mathrm{g}$ of sugar), ordinates (vertical) are probabilities of fly mortality

In this study, four insecticides from different chemical classes were examined when applied alone and in binary mixtures against a susceptible strain of $M$. domestica by feeding bioassays. The toxicity of each substance was found to be increased $\left(\mathrm{LC}_{50}\right.$ of each substance was reduced) in the mixtures compared to that when applied alone,except for the toxicity of chlorfenapyr when applied in combinations with fipronil at the fipronil/chlorfenapyr 1:40 ratio and with acetamiprid. The comparison of $\mathrm{LC}_{50}$ values of acetamiprid, when applied alone and in the mixture (Table 2, 3), suggests that there is the potential of extending the use of this insecticide to $M$. domestica management if properly matched with another insecticide in the mixture. For example, in our study, the acetamiprid/fipronil or acetamiprid/ivermectin combinations had a synergistic effect.
According to our results, ivermectin might be promising for use in mixtures since it had a potentiating effect on other substances in the combinations. The 1:10 ivermectin/chlorfenapyr mixture had the greatest potentiating action of these insecticides on each other and consequently the greatest synergistic effect compared to that of other mixtures. Despite the fipronil/chlorfenapyr mixture at 1:4 ratio being the most toxic of all combinations to flies, its synergistic effect was weak $(\mathrm{CI}=0.8)$.

In our experiments, the interaction patterns (synergistic or antagonistic) of insecticides in the binary mixtures depended on the insecticide combination. For instance, ivermectin/acetamiprid or ivermectin/chlorfenapyr combinations had a synergistic effect, while the interaction between ivermectin and fipronil was antagonistic. According to Jonker et al. (2005) for binary mixtures, one can observe an antagonism or synergism 
depending on whether the toxicant 1 or the toxicant 2 is the main cause of toxicity of the mixture. Most often the synergism of insecticides (usually pyrethroids and organophosphates) is associated with the activity of detoxification of enzymes systems in insects. Khan et al. (2013b) reported the synergistic interaction in mixtures between pyrethroids and organophosphates and explained this effect based on literature data. Briefly, organophosphates hypothetically impact on the degradation speed of pyrethroids by monooxygenase enzymes due to the competition between organophosphates and pyrethroids to binding with monooxygenases. Monooxygenases are the largest and the most important in the detoxification enzyme family, and they take part in degradation of neonicotinoids, fipronil, and avermectins in insects (Markussen \& Kristensen, 2010; David et al., 2013; Scott et al., 2014). At the same time, chlorfenapyr is converted by monooxygenases into an active compound that is able to uncouple the oxidative phosphorylation in insect cells (Black et al., 1994). We proposed that the chlorfenapyr toxicity in binary mixtures would depend on the affinity of another insecticide in the mixture to monooxygenases. In this context, probably, there was the competition between insecticides we tested to binding with these enzymes much as described for pyrethroids and organophosphates.
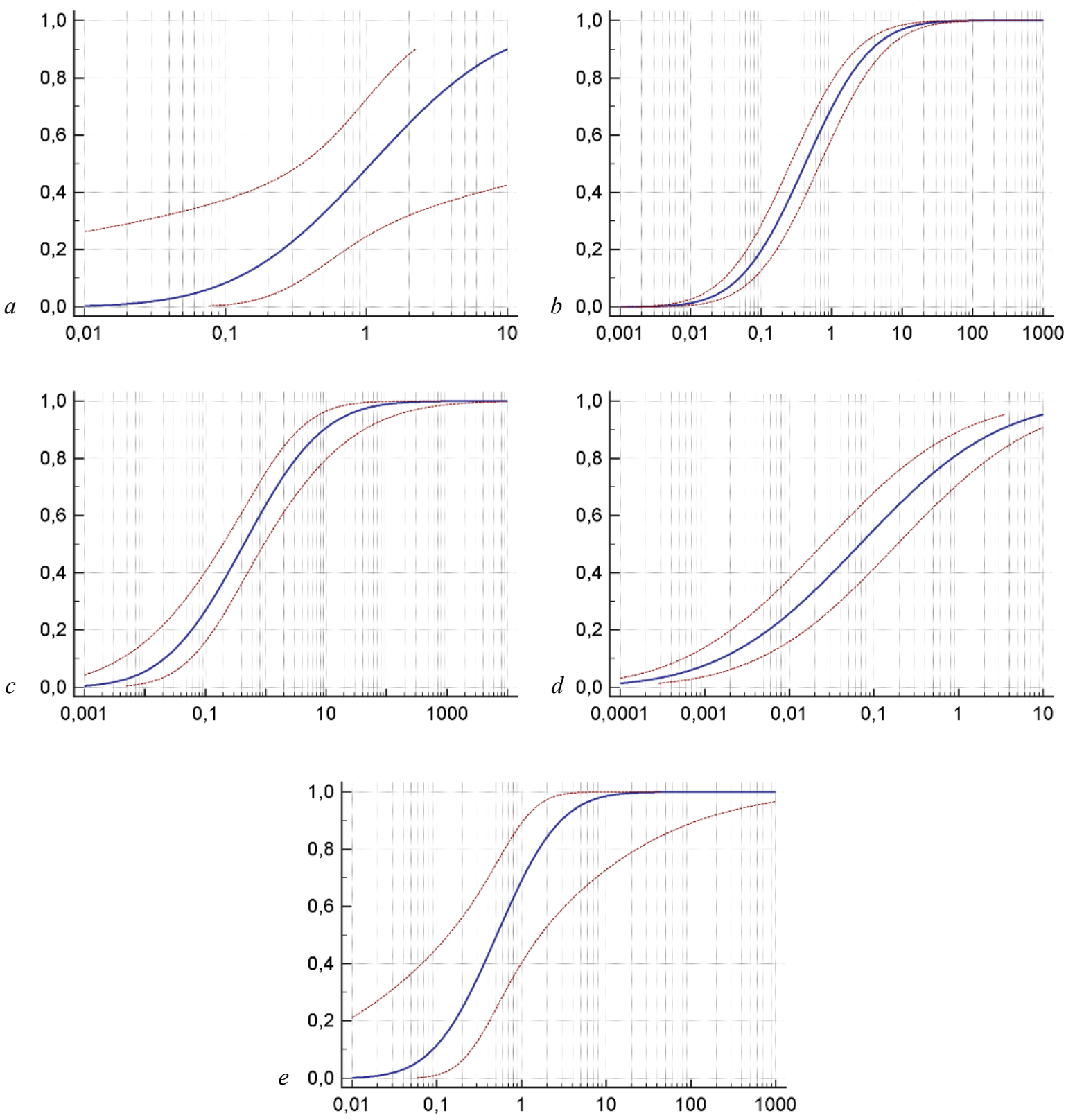

Fig. 4. Dose-response plots (blue line) with confidence intervals (red lines) for ivermectin tested alone ( $a$ ) and in the ivermectin/acetamiprid ( $b$ ), ivermectin/chlorfenapyr $(1: 3)(c)$, ivermectin/chlorfenapyr $(1: 10)(d)$, and fipronil/ivermectin $(e)$ mixtures against adults of Musca domestica in the laboratory no-choice feeding bioassay: abscissae (horizontal) are doses ( $\mathrm{g} / \mathrm{g}$ of sugar), ordinates (vertical) are probabilities of fly mortality

According to our results, the interaction patterns of insecticides in the mixtures depended on the ratio of active ingredients as well. For instance, for the mixtures of fipronil and chlorfenapyr, there was an antagonism at the fipronil/chlorfenapyr 1:40 ratio and the close to additive effect at the fipronil/chlorfenapyr 1:4 ratio. Literature data confirmed the active ingredient ratios and the impact of their concentrations on the interaction pattern of insecticides in the mixtures. Corbel et al. (2003) noted the decrease of synergism when concentrations of propoxur and permethrin increased in the mixture (Corbelet al., 2003). According to Khan et al. (2013b), CI values of mixtures of fipronil and pyrethroids and the kind of interaction between insecticides were different depending on active ingredient ratios. For example, the 1:1 mixture of deltamethrin and fipronil had an antagonistic effect $(\mathrm{CI}=3.88)$ and the 1:6.79 mixture of fipronil and deltamethrin gave synergistic effect $(\mathrm{CI}=$ 0.39 ) against the laboratory susceptible strain of $M$. domestica (Khan et al., 2013b). Abbas et al. (2015) reported that mixtures of emamectin (that 
belongs to avermectins) with profenofos or lambda-cyhalothrin had antagonistic effect against the susceptible strain of $M$. domestica. According to the results by Abbas et al. (2015), CI values of these mixtures decreased when insecticide ratios changed from 1:1 to 1:20 and emamectin concentrations increased.

$a$
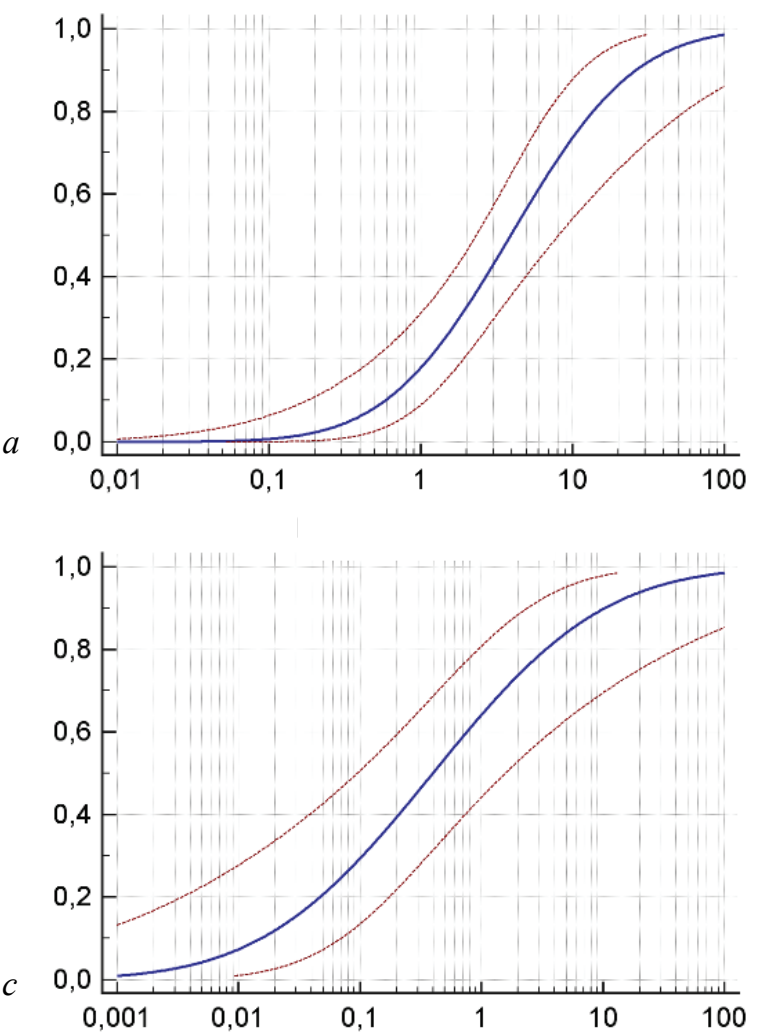

$b$

At the same time, Khan et al. (2013b) found that interactions between emamectin and other pyrethroids (bifenthrin, deltamethrin, cypermethrin) in binary mixtures against $M$. domestica were synergistic or close to additive and CI values of these mixtures depended on active ingredient ratios as well.
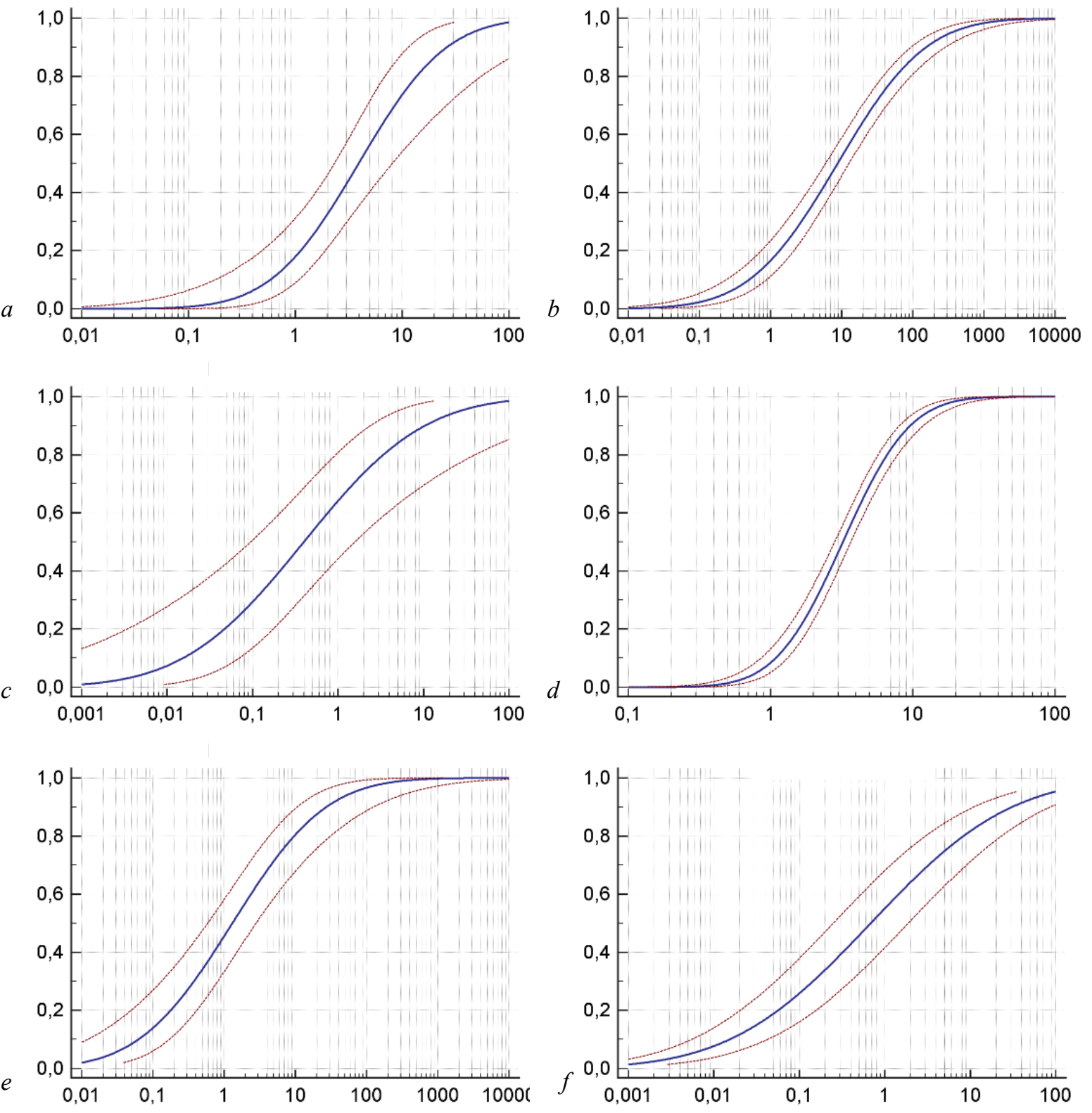

Fig. 5. Dose-response plots (blue line) with confidence intervals (red lines) for chlorfenapyr tested alone $(a)$ and in the acetamiprid/chlorfenapyr $(b)$, fipronil/chlorfenapyr (1:4) $(c)$, fipronil/chlorfenapyr (1:40) $(d)$, ivermectin/chlorfenapyr(1:3) $(e)$, and ivermectin/chlorfenapyr(1:10) $(f)$ mixtures against adults of Musca domestica in the laboratory no-choice feeding bioassay: abscissae (horizontal) are doses $(\mu \mathrm{g} / \mathrm{g}$ of sugar), ordinates (vertical) are probabilities of fly mortality

There are questions about an explanation of the impact of insecticide concentrations and the ratio of active ingredients on their interaction pattern in mixtures. Generally, synergistic or antagonistic effects of binary mixtures may be associated with several causes. First, detoxification degrees of mixture compounds are different in living organisms and achieving the target sites by different compounds takes different amounts of time (Eremina \& Ibragimkhalilova, 2010). Next, the chemical structure of compounds could change the toxicological effect of mixtures like this as has been suggested for fipronil (Taillebois \& Thany, 2016). Finally, compounds of mixtures may have dose-dependent secondary effects, particularly in living organisms, resulting inchanges in the toxicological effect of mixtures (Ritz \&Streibig, 2014).

\section{Conclusion}

Summing up, our results showed that the interaction patterns (synergistic or antagonistic) of insecticides in the binary mixtures depended on (1) the insecticide combination and on (2) the ratio of active ingredients. The combinations of acetamiprid with chlorfenapyr and fipronil with ivermectin or chlorfenapyr (1:40) had an antagonistic effect on M. domestica mortality. The 1:4 fipronil/chlorfenapyr mixture was the more toxic to adults of $M$. domestica, and the ivermectin/chlorfenapyr (preferable at the ivermectin/chlorfenapyr ratio 1:10) or ivermectin/acetamiprid mixtures gave the highest synergistic effects. Thus, these insecticide combinations are more interesting for future evaluation for use against field populations of M. domestica with the aim of designing a new insecticide formulation. 

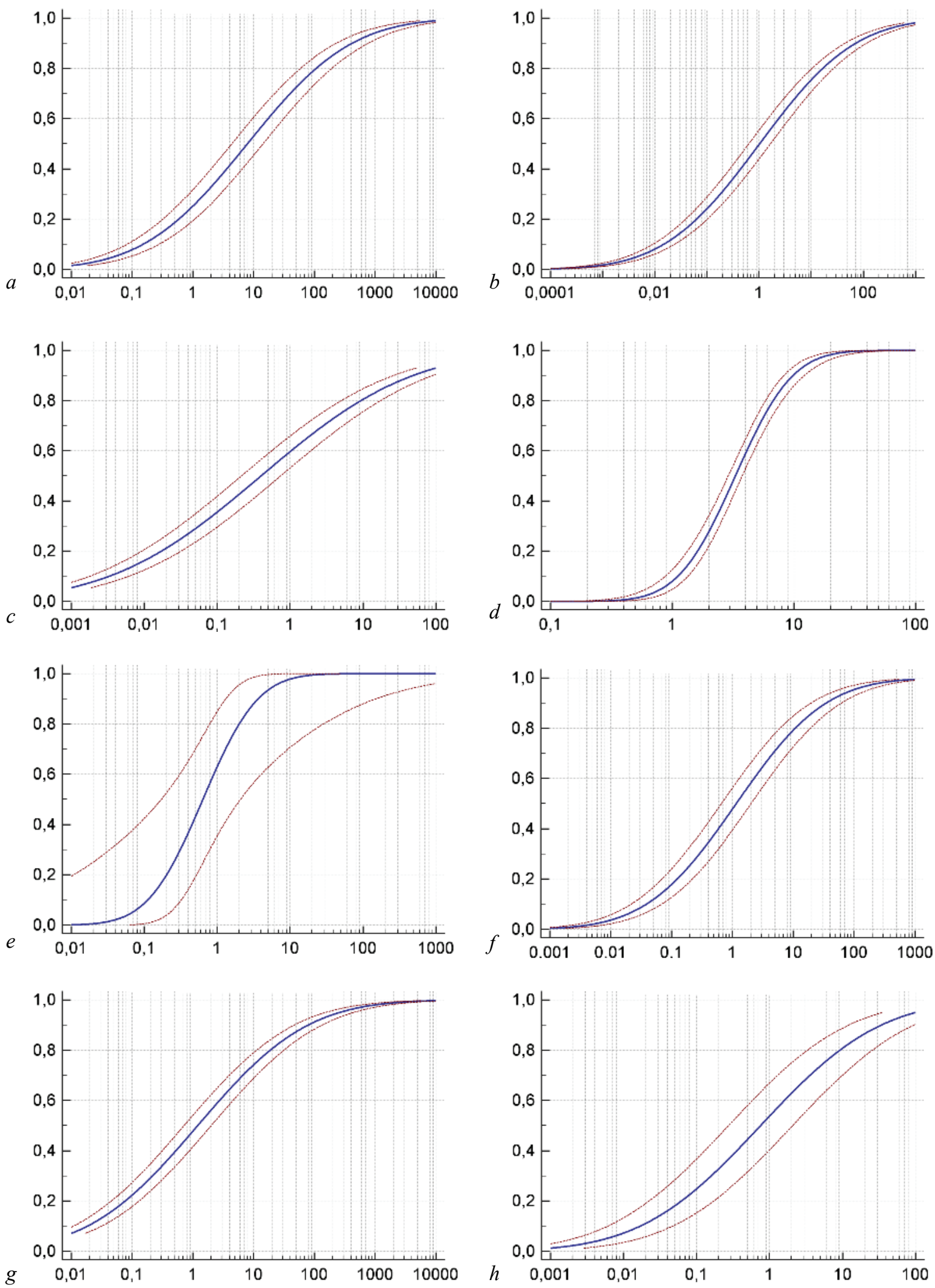

Fig. 6. Dose-response plots (blue line) with confidence intervals (red lines) for the acetamiprid/chlorfenapyr (a), fipronil/acetamiprid $(b)$, fipronil/chlorfenapyr (1:4) $(c)$, fipronil/chlorfenapyr (1:40) $(d)$, fipronil/ivermectin $(e)$, ivermectin/acetamiprid $(f)$, ivermectin/chlorfenapyr $(1: 3)(g)$, and ivermectin/chlorfenapyr(1:10) $(h)$ mixtures against adults of Musca domestica in the laboratory no-choice feeding bioassay: abscissae (horizontal) are doses ( $\mu \mathrm{g} / \mathrm{g}$ of sugar), ordinates (vertical) are probabilities of fly mortality

This work was supported by the Basic Research Program of the Russian Academy of Sciences (Project "Development of means for disinsectization of objects of veterinary supervision"), and was carried out according to the research plan of ASRIVEA - Branch of Tyumen Scientific Centre SB RAS.

\section{References}

Abbas, N., Crickmore, N., \& Shad, S. A. (2015). Efficacy of insecticide mixtures against a resistant strain of house fly (Diptera: Muscidae) collected from a poultry farm. International Journal of Tropical Insect Science, 35(1), 48-53. 
Acevedo, G. R., Zapater, M., \& Toloza, A. C. (2009). Insecticide resistance of house fly, Musca domestica (L.) from Argentina. Parasitology Research, 105, 489-493.

Ahmad, M., Saleem, M. A., \& Sayyed, A. H. (2009). Efficacy of insecticide mixtures against pyrethroid- and organophosphate-resistant populations of Spodoptera litura (Lepidoptera: Noctuidae). Pest Management Science, 65(3), 266-274.

Bass, C., Denholm, I., Williamson, M. S., \& Nauen, R. (2015). The global status of insect resistance to neonicotinoid insecticides. Pesticide Biochemistry and Physiology, 121, 78-87.

Black, B. C., Hollingworth, R. M., Ahammadsahib, K. I., Kukel, C. D., \& Donovan, S. (1994). Insecticidal action and mitochondrial uncoupling activity of AC-303,630 and related halogenated pyrroles. Pesticide Biochemistry and Physiology, 50, 115-128.

Casida, J. E., \& Durkin, K. A. (2013). Neuroactive insecticides: Targets, selectivity, resistance, and secondary effects. Annual Review of Entomology, 58, 99-117.

Cloyd, R. A., \& Raudenbush, A. L. (2014). Efficacy of binary pesticide mixtures against western flower thrips. Horttechnology, 24(4), 449-456.

Corbel, V., Chandre, F., Darriet, F., Lardeux, F., \& Hougard, J. M. (2003). Synergism between permethrin and propoxur against Culex quinquefasciatus mosquito larvae. Medical and Veterinary Entomology, 17, 158-164

David, J. P., Ismail, H. M., Chandor-Proust, A., \& Paine, M. J. I. (2013). Role of cytochrome P450s in insecticide resistance: Impact on the control of mosquito-borne diseases and use of insecticides on Earth. Philosophical Transactions of the Royal Society B: Biological Science, 368, 20120429.

Doud, C. W., Scott, H. M., \& Zurek, L. (2014). Role of house flies in the ecology of Enterococcus faecalis from wastewater treatment facilities. Microbial Ecology, 67(2), 380-391.

Durel, L., Estrada-Peña, A., Franc, M., Mehlhom, H., \& Bouyer, J. (2015). Integrated fly management in European ruminant operations from the perspective of directive 2009/128/EC on sustainable use of pesticides. Parasitology Research, 114, 379-389.

Eremina, O. Y., \& Ibragimkhalilova, I. V. (2010). Synergistic effect of binary neonicotinoid-pyrethroid mixtures on insects. Agrokhimiya, 2, 37-44.

Jonker, M. J., Svendsen, C., Bedaux, J. J., Bongers, M., \& Kammenga, J. E. (2005). Significance testing of synergistic/antagonistic, dose level-dependent, or dose ratio-dependent effects in mixture dose-response analysis. Environmental Toxicology and Chemistry, 24(10), 2701-2713.

Kaufman, P. E., Nunez, S. C., Mann, R. S., Geden, C. J., \& Scharf, M. E. (2010). Nicotinoid and pyrethroid insecticide resistance in houseflies (Diptera: Muscidae) collected from Florida dairies. Pest Management Science, 66, 290-294.

Kaufman, P. E., Scott, J. G., \& Rutz, D. A. (2001). Monitoring insecticide resistance in house flies (Diptera: Muscidae) from New York dairies. Pest Management Science, 57(6), 514-521.

Khamesipour, F., Lankarani, K., Honarvar, B., \& Kwenti, T. (2018). A systematic review of human pathogens carried by the housefly (Musca domestica L.). BMC Public Health, 18, 1049.

Khan, H. A. A., Akram, W., Shad, S. A., \& Lee, J. J. (2013b). Insecticide mixtures could enhance the toxicity of insecticides in a resistant dairy population of Musca domestica L. PLoS One, 8(4), e60929.

Khan, H. A., Akram, W., \& Shad, S. A. (2013a). Resistance to conventional insecticides in Pakistani populations of Musca domestica L. (Diptera: Muscidae): A potential ectoparasite of dairy animals. Ecotoxicology, 22, 522-527.

Malik, A., Singh, N., \& Satya, S. (2007). House fly (Musca domestica): A review of control strategies for a challenging pest. Journal of Environmental Science and Health, Part B, 42, 453-469.
Markussen, M. D. K., \& Kristensen, M. (2010). Cytochrome P450 monooxygenasemediated neonicotinoid resistance in the house fly Musca domestica L. Pesticide Biochemistry and Physiology, 98, 50-58.

Markussen, M. D. K., \& Kristensen, M. (2011). Spinosad resistance in female Musca domestica L. from a field-derived population. Pest Management Science, 68, 75-82.

Mohammed, A. N., Abdel-Latef, G. K., Abdel-Azeem, N. M., \& El-Dakhly, K. M. (2016). Ecological study on antimicrobial-resistant zoonotic bacteria transmitted by flies in cattle farms. Parasitology Research, 115(10), 3889-3896.

Nasir, M., Imran, M., \& Ahmad, M. (2013). Pyrethroids synergize new chemical insecticides in field populations of Plutella xylostella (Lepidoptera: Plutellidae). Pakistan Journal of Zoology, 45(3), 629-633.

Nazari, M., Mehrabi, T., Hosseini, S. M., \& Alikhani, M. Y. (2017). Bacterial Contamination of adult house flies (Musca domestica) and sensitivity of these bacteria to various antibiotics, captured from Hamadan City, Iran. Journal of Clinical and Diagnostic Research, 11, DC04-DC07.

Ngufor, C., Critchley, J., Fagbohoun, J., N'Guessan, R., Todjinou, D., \& Rowland, M. (2016). Chlorfenapyr (a pyrrole insecticide) applied alone or as a mixture with alpha-cypermethrin for indoor residual spraying against pyrethroid resistant Anopheles gambiae Sl: An experimental hut study in Cove, Benin. PLoS One, 11, e0162210.

Omura, S., \& Crump, A. (2004). The life and times of ivermectin - a success story. Nature Reviews Microbiology, 2(12), 984-989.

Ritz, C., \& Streibig, J. C. (2014). From additivity to synergism: A modelling perspective. Synergy, 1, 22-29.

Scott, J. G., Leichter, C. A., Rinkevich, F. D., Harris, S. A., Su, C., Aberegg, L. C Moon, R., Geden, C. J., Gerry, A. C., Taylor, D. B., Byford, R. L., Watson, W., Jonson, G., Boxler, D., \& Zurek, L. (2013). Insecticide resistance in house flies from the United States: Resistance levels and frequency of pyrethroid resistance alleles. Pesticide Biochemistry and Physiology, 107, 377-384.

Scott, J. G., Warren, W. C., Beukeboom, L. W., Bopp, D., Clark, A. G., Giers, S. D., Hediger, M., Jones, A. K., Kasai, S., Leichter, C. A. (2014). Genome of the house fly, Musca domestica L., a global vector of diseases with adaptations to a septic environment. Genome Biology, 15, 466.

Sparks, T. C., \& Nauen, R. (2015). IRAC: Mode of action, classification and insecticide resistance management. Pesticide Biochemistry and Physiology, 121, 122-128.

Sudo, M., Takahashi, D., Andow, D. A., Suzuki, Y., \& Yamanaka, T. (2017) Optimal management strategy of insecticide resistance under various insect life histories: Heterogeneous timing of selection and interpatch dispersal. Evolutionary Applications, 11(2), 271-283.

Taillebois, E., \& Thany, S. H. (2016). The differential effect of low-dose mixtures of four pesticides on the pea aphid Acyrthosiphon pisum. Insects, 7(4), 53

Usui, M., Shirakawa, T., Fukuda, A., \& Tamura, Y. (2015). The role of flies in disseminating plasmids with antimicrobial-resistance genes between farms. Microbial Drug Resistance, 21, 562-569.

Wang, Y. C., Chang, Y. C., Chuang, H. L., Chiu, C. C., Yeh, K. S., Chang, C. C., Hsuan, S. L., Lin, W. H., \& Chen, T. H. (2011). Transmission of Salmonella between swine farms by the housefly (Musca domestica). Journal of Food Protection, 74, 1012-1016.

Yuan, J. Z., Li, Q. F., Huanga, J. B., \& Gao, J. F. (2015). Effect of chlorfenapyr on cypermethrin-resistant Culex pipiens pallens Coq mosquitoes. Acta Tropica, $143,13-17$.

Zhu, F., Lavine, L., O’Neal, S., Lavine, M., Foss, C., \& Walsh, D. (2016). Insecticide resistance and management strategies in urban ecosystems. Insects, 7, 2. 